\title{
Management of low and high grade squamous intraepithelial lesions during pregnancy
}

\author{
Panagakis G., Theofanakis H, Pappa K, Loutradis D. \\ Athens, Athens, Greece \\ Correspondence \\ Georgios Panagakis ,MD, 76-78, Kaukasou st.,11363 Athens, Greece, email:gpssas@yahoo.gr
}

1st Department of Obstetrics and Gynecology Alexandra hospital, National and Kapodistrian University of

Key words: CIN, LGSIL, HGSIL, pregnancy

\section{Introduction}

Despite significant advances regarding screening and treatment of cervical dysplasia, cervical cancer in fertile age is becoming more and more common in the last two decades ${ }^{1}$. Cervical cancer screening ideally detects preinvasive lesions that can be eradicated or early stage cervical cancer that can be successfully treated. Cervical cancer screening was previously limited to cervical cytology. However, during the past decade, High-Risk Human Papillomavirus (HR HPV) testing has also become an important screening tool ${ }^{2}$. The incidence of cervical cancer in pregnancy is estimated to be $1-10 / 10000$ pregnancies ${ }^{3}$, while abnormal cervical cytology is at least as high as reported for non- pregnant women ${ }^{4}$. Among dysplasia cases diagnosed during pregnancy, 10\%-70\% regress and sometimes even disappear postpartum, while persistence in the severity of cervical neoplasia is reported in $25 \%-47 \%$ and progression occurs in 3\%-30\%. However, adequate follow-up and definitive management in the postpartum period is important ${ }^{3}$. There are various strategies in different countries whether to perform cervical screening in pregnancy. Differences depend on variables such as local incidence of cervical can- cer, presence of organized screening in each country, population coverage by screening, social awareness of the disease, etc. However, there are no medical reasons why screening should not be performed during pregnancy. The objective of this review is to assess proper management of Squamous Intraepithelial Lesions (SIL) during pregnancy.

\section{Screening tolls \\ Cervical cytology}

Cervical cytology became the standard screening test for cervical cancer and premalignant cervical lesions with the introduction of the Papanicolaou (Pap) smear in 19415. Liquid-based, thin-layer preparation of cervical cytology specimens was a subsequent modification of this technique. Both test modalities detect most cases of cervical neoplasia during the typically prolonged premalignant or early occult invasive phases, when treatment outcomes are optimal. Both of them are considered equally acceptable for screening ${ }^{6}$. Terminology for reporting cervical cytology was standardized by the Bethesda System in 19887. This system has been revised several times, and the current system was de- 
veloped in $2014^{8}$.

\section{HPV testing}

The HPV test is primarily used to screen for cervical cancer and/or identify women who may be at increased risk of the disease. The test determines whether a woman's cervical cells are infected with a high-risk type of HPV (hrHPV). The HPV test detects the virus, but not cell changes. A role for HPV testing in cervical cancer screening is attractive due to its improved sensitivity for CIN 3 or cervical cancer and the objectivity of its results. The combination of HPV testing with cytology increases the sensitivity of a single screening test for high-grade neoplasia to nearly 100 $\%$ and leads to earlier detection and management of high-grade lesions (HSIL) ${ }^{9}$.

\section{The Bethesda system}

The Bethesda system (TBS) is a system for reporting cervical or vaginal cytologic diagnoses, used for reporting Pap smear results. It was introduced in 1988, and revised in 1991, 2001 and 2014. The name comes from the location (Bethesda, Maryland) of the conference that established the system ${ }^{10}$.

Abnormal results include :

- Atypical squamous cells

- Atypical squamous cells of undetermined significance (ASC-US)

- Atypical squamous cells - cannot exclude HSIL (ASC-H)

- Low grade squamous intraepithelial lesion (LGSIL or LSIL)

- High grade squamous intraepithelial lesion (HGSIL or HSIL)

- Squamous cell carcinoma

- Atypical Glandular Cells not otherwise specified (AGC-NOS)

- Atypical Glandular Cells, suspicious for AIS or cancer (AGC-neoplastic)

- Adenocarcinoma in situ (AIS)
- Adenocarcinoma

\section{Management of Igsil in pregnancy}

Low-grade squamous intraepithelial lesion (LSIL) is a type of cervical dysplasia that can be seen on a Pap smear. Cervical dysplasia refers to alterations of the cellular characteristics of the cervix as a result of one or more factors. LSIL is considered a mild form of cervical dysplasia involving the presence of early changes in the morphology, size and shape of the cervical cells. The appearance of cervical dysplasia refers to the development of pre-cancerous tumors in the cervix. It carries a $15-30 \%$ risk of CIN2 or $3^{2}$. Prevalence of Cervical Intraepithelial Lesion (CIN) in pregnancy is relatively high from 0,19 to $6,7 \%$. Majority of CIN1 constitute up to $86 \%$ of all CIN cases $^{11}$. Studies report that $10 \%-70 \%$ of dysplasia cases diagnosed during pregnancy regress and sometimes even disappear postpartum, while persistence in the severity of cervical neoplasia is reported in $25 \%$ $47 \%$ of cases and progression in 3\%-30\% of cases ${ }^{3}$. It has been found that pregnant patients with ASCUS or LSIL cytology rarely have colposcopically suspected CIN 2,3 at their initial colposcopy that warrants a cervical biopsy ${ }^{12}$. In pregnant women with LGSIL, the risk of invasive cancer is considered very low, but colposcopy should be performed. If colposcopy shows no signs of invasion, no biopsy should be performed. In cases where colposcopy is consistent with cytology results, no further followup during pregnancy is needed and no treatment should be implemented. There is also no oncological indication for cesarean section. Biopsy is recommended if invasive disease is suspected. Otherwise patient should be referred for cytology 6-8 weeks after delivery ${ }^{13,14}$.

\section{Management of hgsil in pregnancy}

High-grade squamous intraepithelial lesion (HSIL) is a squamous cell abnormality associated with HPV. 
It encompasses the previously used terms of CIN2, CIN3, moderate and severe dysplasia and carcinoma in situ. This current terminology for HSIL was introduced by the Bethesda System for Reporting Cervical Cytology (TBS) for cytology specimens in 1988, and has been adopted since, for histology specimens by the Lower Anogenital Squamous Terminology Standardization Consensus Conference (LAST) and the World Health Organization (WHO) in 2012 and 2014, respectively. Though not all HSIL will progress to cancer, it is considered a pre-cancerous lesion and therefore is usually treated aggressively. An increasing incidence of high-grade squamous intraepithelial lesion (HSIL) has been observed among young women. An increased number of cases are being discovered during pregnancy. Controversies exist on the effect of pregnancy on HPV infection. Some studies suggest the possibility that the physiological process of pregnancy modifies certain characteristics of the mother, increasing the risk of both infection and persistence of infection with HPV. Whether pregnancy has an influence on the course of the neoplasia remains unsolved. Studies report that $10 \%-70 \%$ of dysplasia cases diagnosed during pregnancy regress and sometimes even disappear postpartum, while persistence in the severity of cervical neoplasia is reported in $25 \%-47 \%$ of cases and progression in 3\%$30 \%$ of cases $^{3}$

The indications for colposcopy in pregnant women are essentially the same as in non-pregnant women. If HSIL is found, evaluation with colposcopy is essential ${ }^{15}$. Cervical biopsies can be performed during pregnancy, while whether the LEEP influences pregnancy outcome is still debatable. Cervical conization during pregnancy is associated with a significant morbidity for both mother and fetus. In general, pregnant women found to have HSIL cytology should not undergo excisional treatment; only colposcopy is acceptable. If a histologic diagnosis of a high-grade lesion is made, additional cytologic and colposcopic exams may be performed up to every 12 weeks. If cytology results are suggestive of invasive cancer or if the lesions progress, a repeat biopsy is recommended. It is also considered acceptable to defer re-evaluation until the patient is at least six weeks postpartum. A diagnostic excisional procedure is only recommended if there is a concern for invasive cancer ${ }^{16}$. The European Society of Gyneacological Oncology (ESGO) suggests that colposcopy should be performed with no undue delay. When colposcopy suggests CIN1 or less, biopsy is not recommended. When colposcopy suggests CIN2 or 3 biopsies may be obtained. When colposcopy shows signs of invasion (e.g. atypical vessels), excisional biopsy is recommended. If there is no invasion diagnosed, patient should be referred to cytology and repeat diagnostic process 6-8 weeks after delivery. During pregnancy additional cytology and colposcopy may be performed after 12 weeks as followup. Repeated biopsy may be performed only if colposcopy or cytology indicate invasive cancer. CIN2 or 3 should not be treated during pregnancy, if invasion is excluded. As far as the mode of delivery is concerned, there is no oncological indication for cesarean section in preinvasive lesions ${ }^{17}$.

\section{Conclusion}

Current data on the spontaneous evolution of an intraepithelial neoplasia during pregnancy are quite diverse but ESGO notes that prevalence of CIN in pregnancy is relatively high from 0,19 to $6,7 \%$. Majority of CIN1 constitute up to $86 \%$ of all CIN cases. Prevalence of CIN3 is $0,1-0,2 \%$ in pregnant women. Regarding LSIL, colposcopy should be performed and if it is consistent with cytology results, no further follow-up during pregnancy is needed and no treatment should be implemented. Cytology should be repeated 6-8 weeks postpartum. In cases of HSIL, colposcopy is very important in order to exclude invasive disease and it should be performed as soon as 
possible. Biopsies should be taken only if CIN2 or 3 is suspected and if there is no invasion present, patient should be referred to perform cytology and repeat diagnostic process 6-8 weeks after delivery. However, follow up with both cytology and colposcopy should be performed after 12 weeks. For both types of preinvasive disease, adequate followup and definitive management in the postpartum period is important.

\section{References}

1. Maneo A, Sideri M, Scambia G et al. Simple conization and lymphadenectomy for the conservative treatment of stage IB1 cervical cancer. An Italian experience. GynecolOncol 2011. 123(3): 557-60.

2. Williams Gynecology. 3rd ed. 2016: McGraw-Hill.

3. Frega A, Scirpa P, Corosu R, Verrico M, Scarciglia ML,et al. Clinical management and follow-up of squamous intraepithelial cervical lesions during pregnancy and postpartum. Anticancer Res 2007. 27(4C): 2743-6.

4. Williams Obstetrics. 24th ed. 2014: McGraw-Hill.

5. Papanicolaou, GN, Traut HF. The diagnostic value of vaginal smears in carcinoma of the uterus. 1941. Arch Pathol Lab Med 1997. 121(3): 211-24.

6. Committee on Gynecologic, P. Committee opinion No. 534: well-woman visit. ObstetGynecol, 2012. 120(2 Pt 1): 421-4.

7. Nayar R, Wilbur DC. The Pap test and Bethesda 2014. Cancer Cytopathol 2015. 123(5):271-81.

8. Nayar R, Wilbur DC. The Pap Test and Bethesda 2014. "The reports of my demise have been greatly exaggerated." (after a quotation from Mark Twain). ActaCytol 2015. 59(2): 121-32.

9. Ronco G, Giorgi-Rossi P, Carozzi F, Confortini M, Dalla Palma Pet al. Efficacy of human papillomavirus testing for the detection of invasive cervical cancers and cervical intraepithelial neoplasia: a randomised controlled trial. Lancet
Oncol 2010. 11(3): 249-57.

10. Nayar R, Solomon D. Second edition of 'The Bethesda System for reporting cervical cytology' - atlas, website, and Bethesda interobserver reproducibility project. Cytojournal 2004.1(1): 4.

11. Freeman-Wang T, Walker P. The management of cervical pre-malignancy and malignancy in pregnancy, in The Cervix, J.A. Jordan and A. Singer, Editors. 2006, Blackwell Publishing.

12. Wetta LA, Matthews KS, Kemper ML, Whitworth JM, Fain ET, Huh WK et al. The management of cervical intraepithelial neoplasia during pregnancy: is colposcopy necessary? J Low Genit Tract Dis, 2009. 13(3): 182-5.

13. Wright TC Jr, Massad LS, Dunton CJ, Spitzer M, Wilkinson EJ, Solomon D et al. 2006 consensus guidelines for the management of women with cervical intraepithelial neoplasia or adenocarcinoma in situ. J Low Genit Tract Dis 2007. 11(4): 223-39.

14. Wright TC Jr, Massad LS, Dunton CJ, Spitzer M, Wilkinson EJ, Solomon D et al. 2006 consensus guidelines for the management of women with cervical intraepithelial neoplasia or adenocarcinoma in situ. J Low Genit Tract Dis 2007. 11(4): 201-22.

15. Bentley J, Executive Council Of The Society of Canadian and Special Contributors. Colposcopic management of abnormal cervical cytology and histology. J ObstetGynaecol Can 2012. 34(12): 1188-1202.

16. Khieu M and Butler SL. Cancer, Squamous Cell, High Grade Squamous Intraepithelial Lesion (HGSIL), in StatPearls 2017: Treasure Island (FL).

17. Kolawa W, Nieminen P. Cervical Intraepithelial Neoplasia (CIN), in Textbook of Cancer in Pregnancy, F. Amantet al. 2017, ESGO.

Received 8-4-2018

Revised 2-5-2018

Accepted 21-5-2018 